\title{
An Improved Approach to Passive Testing of FSM-based Systems
}

\author{
Hasan Ural, Zhi Xu and Fan Zhang \\ SITE, University of Ottawa, Ottawa, Ontario, K1N 6N5, Canada \\ \{ural,zxu061, fzhang\}@site.uottawa.ca
}

\begin{abstract}
Fault detection is a fundamental part of passive testing which determines whether a system under test (SUT) is faulty by observing the input/output behavior of the SUT without interfering its normal operations. In this paper, we propose a new approach to Finite State Machine (FSM)-based passive fault detection which improves the performance of the approach in [4] and gathers more information during testing compared with the approach in [4]. The results of theoretical and experimental evaluations are reported.
\end{abstract}

\section{Introduction}

Passive fault detection is a testing technique used in fault management of a system under test by observing its input/output behaviors without interfering its normal operations [5]. In Finite State Machine (FSM)based passive fault detection, the specification of the system under test (SUT) is modeled as an FSM $M$, SUT $N$ is treated as a black-box FSM, and the tester wishes to determine whether $N$ is faulty with respect to $M$ by observing a sequence $Q$ of I/O pairs from $N$ where the starting state (when $Q$ starts) of $N$ is unknown. Such a decision can be based on the number of states that are compatible with $Q$. A state $s$ of $M$ is compatible with $Q$ if $Q$ is a trace of $M$ starting at $s$. If the number of states compatible with $Q$ is zero then $Q$ is sufficient to determine that $N$ is faulty. Otherwise, $Q$ is insufficient to determine whether $N$ is faulty. That is there are one or more states compatible with $Q$ and $Q$ needs to be augmented by an additional I/O sequence of $N$ to continue with the fault detection.

Lee et al developed algorithms for FSM-based passive fault detection [4]. Their approach can be summarized as follows: suppose that the starting state of $N$ is any state of $M$, check the observed sequence $Q$ of I/O pairs one-by-one from the beginning, reduce the size of the set $S^{\prime}$ of possible current states by eliminating impossible states until either $S^{\prime}$ is empty ( $N$ is faulty) or there is at least one state in $S^{\prime}$ (no fault is detected by $Q$ ). This approach has been applied to
FSM-based systems $[10,11,12]$ and has been extended to systems specified in the Extended FSM model by $[1,2,5,6,10]$ and to systems specified in the Communicating FSM model by [7, 8].

The algorithm in [4] is comprehensive but not efficient enough. In this algorithm, every state of $M$ needs to be checked. However, the number of states compatible with $Q$ is usually comparatively small and checking every state of $M$ would be unnecessary. Further, this algorithm only determines the set of possible current states when it terminates. The information about possible starting state and possible trace corresponding to $Q$ is not provided unless a postprocessing is performed. Clearly, the approaches derived from [4] also have these two shortcomings. To improve the efficiency of FSM-based passive fault detection and gather more information during testing, we propose a new approach to FSM-based passive fault detection which is based on the following approach: randomly pick a state $s$ in subset $S_{0}$ of the set of states of $M$ and determine whether $Q$ is the trace of $M$ at $s$. If $s$ is compatible with $Q$, stop and declare that $Q$ is not sufficient to determine whether $N$ is faulty. In this case, $Q$ is a trace of $M$ at $s$ and the current state of $M$ can be determined readily. Otherwise, continue to check other states in $S_{0}$. After checking all the states in $S_{0}$, if no state is found to be compatible with $Q$, then $N$ is declared to be faulty. Note that we took $S_{0}$ to be equal to the set of states of $M$ when we perform analytical and experimental comparisons of the approach we propose with the approach in [4] in an effort not to put the approach in [4] at a disadvantage.

The rest of the paper is organized as follows: Section 2 defines the terms and notations used in the paper. Section 3 describes algorithms for the proposed approach for the FSM-based passive fault detection, and compares the computational complexities of these algorithms. Section 4 presents the results of an experimental evaluation. Section 5 concludes the paper.

\section{Preliminaries}

An $F S M M$ is a quintuple $=(S, X, Y, \delta, \lambda)$, where $S$ 
$=\left\{s_{1}, s_{2}, \ldots, s_{n}\right\}$ is a finite set of states with $n=|S|$ and $s_{1} \in S$ as the initial state, $X$ is a nonempty finite set of inputs, $Y$ is a nonempty finite set of outputs, $\delta$ is a state transition function that maps $S \times X$ to $S$, and $\lambda$ is an output function that maps $S \times X$ to $Y$. These two functions are extended to input sequences $I \in X^{*}$ in the standard manner. The FSM $M$ defined above is deterministic, i.e., if for each input $x \in X$, there is at most one transition defined at each state of $M$.

$M$ can be represented by a directed graph $G=(V, E)$ (Figure 1) where a set of vertices $V=\left\{v_{1}, v_{2}, \ldots, v_{n}\right\}$ represents the set of states of $M$ and a set of edges $E=$ $\left\{\left(v_{j}, v_{k} ; x / y\right): v_{j}, v_{k} \in V\right\}$ represents all specified transitions of $M$, i.e., edge $e=\left(v_{j}, v_{k} ; x / y\right)$ represents a state transition from state $s_{j}$ to $s_{k}$ with input $x \in X$ and output $y$ $\in Y$, and the $\mathrm{I} / \mathrm{O}$ pair $x / y$ is the label of $e$. The label of a path $e_{1} e_{2} \ldots e_{r}, e_{i} \in E, 1 \leq i \leq r$, is the concatenation of the labels of $e_{i}$ and is called an $\mathrm{I} / \mathrm{O}$ sequence. The $\mathrm{I} / \mathrm{O}$ sequence $I / \lambda\left(s_{i}, I\right)$ is called the trace of $M$ at $s_{i}$.

In this paper, we assume that both $M$ and $N$ are deterministic FSMs, an I/O sequence $Q$ is observed from $N$, and a set of possible starting states $S_{0}$ of $M$ is given. We wish to determine whether there is no state $s$ in $S_{0}$ such that $Q$ is a trace of $M$ at $s$.

Example 1. Let $M$ be as in Figure 1 and $S_{0}=\left\{s_{1}, s_{2}\right\}$.

$Q="(\mathrm{a} / 0)(\mathrm{b} / 0)(\mathrm{a} / 0)(\mathrm{b} / 0)(\mathrm{a} / 0)(\mathrm{b} / 0)(\mathrm{b} / 1) "$

$=$ "abababb/0000001". Since "0000001" $=\lambda\left(s_{1}\right.$, abababb), $Q$ is the trace of $M$ at $s_{1}$. Thus, $Q$ is declared to be insufficient to determine whether $N$ is faulty. If $Q$ $="(\mathrm{a} / 0)(\mathrm{b} / 0)(\mathrm{a} / 0)(\mathrm{b} / 0)(\mathrm{a} / 0)(\mathrm{b} / 0)(\mathrm{a} / 1) "=$ "abababa/ 0000001 ", $Q$ is not a trace of $M$ at any state, thus $N$ can be reported to be faulty.

$Q=\left(x_{1} / y_{1}\right)\left(x_{2} / y_{2}\right) \ldots\left(x_{k} / y_{k}\right)$ denotes an I/O sequence of length $k . Q_{j}^{p}$ is the prefix of $Q$ of length $j, Q_{j}^{s}$ is the suffix of $Q$ of length $k-j, 1 \leq j \leq k$.

\section{Algorithms for Passive Fault Detection}

We first present the algorithm proposed by Lee et al [4], then propose three new algorithms for FSM-based passive fault detection. In order to make the analysis and further comparisons of the algorithms, we consider the number of comparisons between the actual output $y_{j}$ and the expected output $\lambda\left(s, x_{j}\right)$ as the measure of computational complexity, $1 \leq j \leq k, s \in S$.

\subsection{The Approach of Lee et al in [4]}

In order to facilitate comparisons, we have rewritten the algorithm given in [4] as Algorithm 0 without changing its computational complexity.

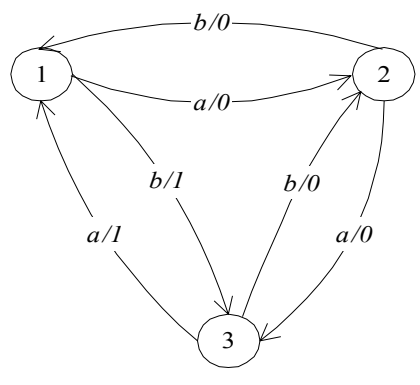

Figure 1. An FSM $M$

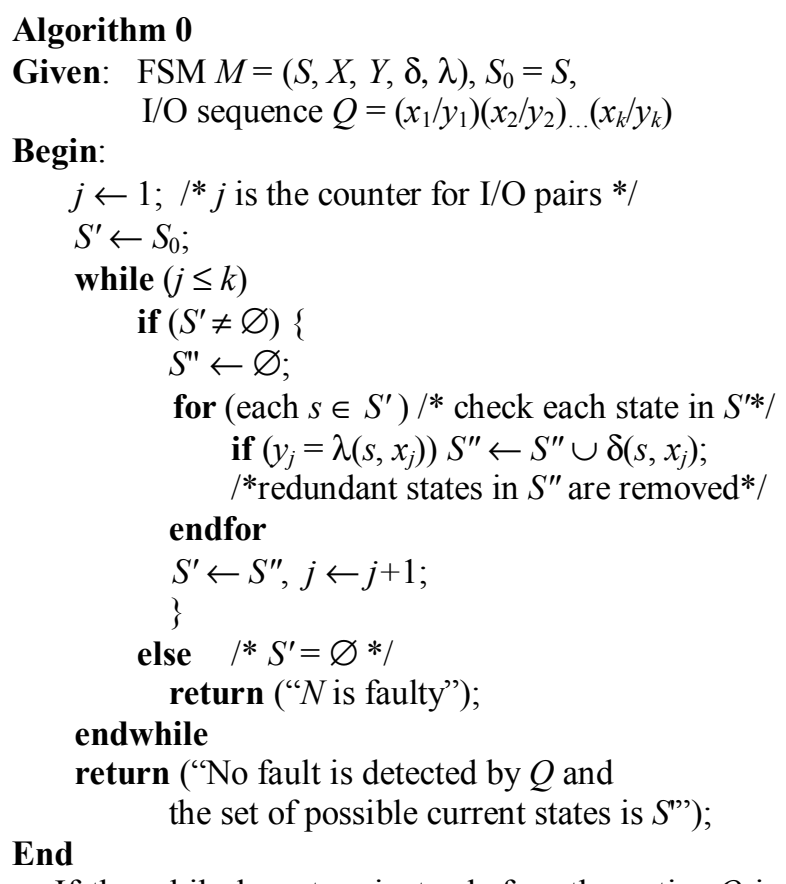

If the while loop terminates before the entire $Q$ is checked, $N$ is declared to be faulty. Otherwise, $Q$ is declared to be insufficient to determine whether $N$ is faulty. In this case, the possible current states are determined but the possible starting states (where $Q$ starts) are unknown. In order to find the set of possible starting states, a post-processing will be needed.

Theorem 1 (Lee et al [4]) Let $S_{j}$ denote the set of possible current states right after the first $j \mathrm{I} / \mathrm{O}$ pairs of $Q$, i.e., $S_{j}=\delta\left(S_{0}, x_{1} x_{2} \ldots x_{j}\right)$. The computational complexity of Algorithm 0 is $C_{1}=\sum_{j=1}^{k}\left|S_{j-1}\right|$.

Proof: In Algorithm 0, every state in the set of possible current states will be checked to compare its related I/O pair with the current I/O pair in $Q$, i.e., for a state $s$ in $S_{j}$, there will be one comparison between $y_{j+1}$ and the expected output $\lambda\left(s, x_{j+1}\right)$, and $\left|S_{j}\right|$ comparisons are needed to check the set $S_{j}$. Thus, the total number of comparisons is $\sum_{j=1}^{k}\left|S_{j-1}\right|$.

Example 2. Applying Algorithm 0 to $M$ (Figure 1) and 
$Q=$ "abababb/0000001", the number of comparisons is $\sum_{j=1}^{k}\left|S_{j-1}\right|=3+2+2+2+2+2+2=15$.

\subsection{The Proposed Approach}

Algorithm 1 is based on our proposed approach which checks, for each state $s \in S_{0} \subseteq S$, whether $Q$ is a trace of $M$ at $s$. It terminates when $Q$ is verified to be a trace of $M$ at a state $s \in S_{0}$ or when all states in $S_{0}$ are checked and no state is found compatible with $Q$.

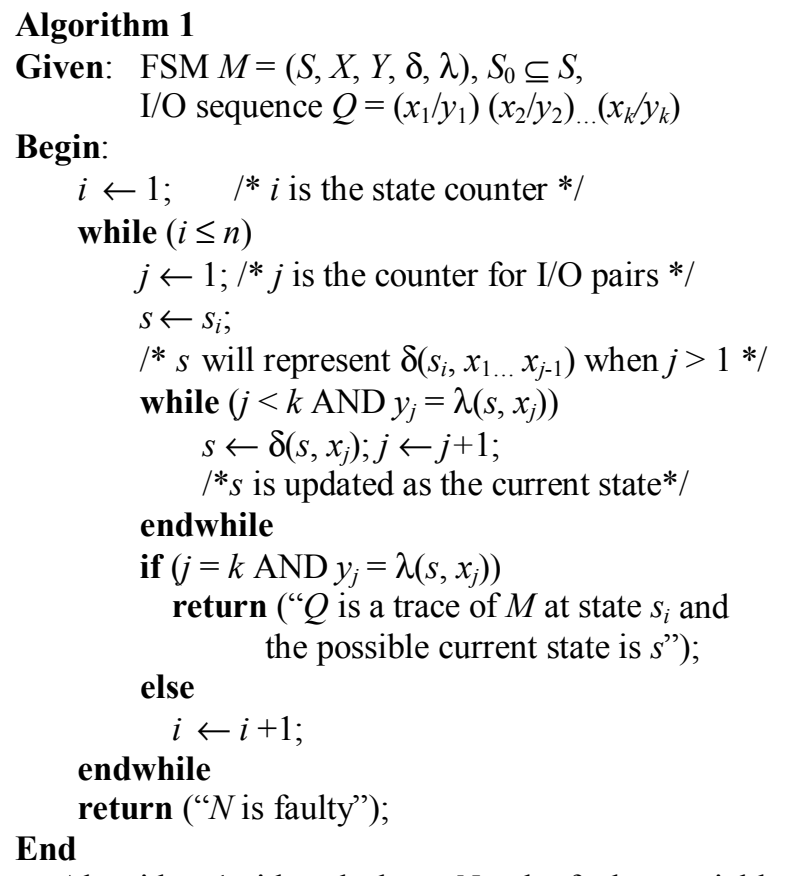

Algorithm 1 either declares $N$ to be faulty or yields both the possible current state $(s)$ and possible starting state $\left(s_{i}\right)$ once a state compatible with $Q$ is found.

Theorem 2 For the given state $s_{i}$ of $M$ and I/O sequence $Q=x_{1} \ldots x_{k} / y_{1} \ldots y_{k}$, let $c_{i}(Q)$ denote the largest number $j(1 \leq j \leq \mathrm{k})$ such that $y_{1} \ldots y_{j-1}=\lambda\left(s_{i}, x_{1} \ldots x_{j-1}\right)$ and $\lambda\left(\delta\left(s_{i}, x_{1} \ldots x_{j-1}\right), x_{j}\right) \neq y_{j}$. Let $C_{2 \text { worst }}\left(M, S_{0}, Q\right)$ $=\sum_{i=1}^{n} c_{i}(Q)$. Let $C_{2}\left(M, S_{0}, Q\right)$ denote the computational complexity of Algorithm 1. If $s_{r}$ is the first state of $M$ such that $Q$ is a trace of $M$ at $s_{r}$, then $C_{2}\left(M, S_{0}, Q\right)$ $=\sum_{i=1}^{r} c_{i}(Q)$; if $N$ is faulty, then $C_{2}\left(M, S_{0}, Q\right)=$ $C_{2 \text { worst }}\left(M, S_{0}, Q\right)=\sum_{i=1}^{n} c_{i}(Q)$.

Proof: In Algorithm 1, each state in $S_{0}$ is checked to determine whether it is compatible with $Q$. The checking procedure for a state $s_{i}$ will not stop until it confronts a mismatch (then the next state $s_{i+1}$ will be selected to check); or the entire sequence $Q$ has been checked and no mismatch found (then $s_{i}$ is reported to be compatible with $Q$ ). The whole checking procedure will terminate when a state compatible with $Q$ is found or when all the states have been checked and no state is found to be compatible with $Q$. Assume $y_{1} \ldots y_{j-1}=$ $\lambda\left(s_{i}, x_{1} \ldots x_{j-1}\right)$ but $y_{j} \neq \lambda\left(\delta\left(s_{i}, x_{1} \ldots x_{j-1}\right), x_{j}\right)$, it means $j$ comparisons (denoted by $c_{i}(Q)$ ) are needed to determine that $Q$ is not a trace of $M$ at $s_{i}$. If $s_{r} \in S_{0}$ is the first state of $M$ such that $Q$ is the trace of $M$ at $s_{r}$, Algorithm 1 will detect mismatch in checking $s_{1} \ldots s_{r-1}$ and stop after checking $s_{r}$. Thus, the total number of comparisons needed is $\sum_{i=1}^{r} c_{i}(Q)$.

Example 3. Applying Algorithm 1 to $M$ (Figure 1) and $Q=$ "abababb/0000001", with $r=1$, the number of comparisons is $C_{2}\left(M, S_{0}, Q\right)=\sum_{i=1}^{r} c_{i}(Q)=c_{1}(Q)=7$.

Algorithm 1 is simple and straightforward, however, it encounters the redundant checking problem which is: two traces starting from different states converge to the same state after applying $Q_{j}^{p}$. In Algorithm 1, the common part $Q_{j}^{s}$ will be rechecked redundantly. In contrast, Algorithm 0 avoids the redundant checking problem by removing redundant states in the set of possible current states.

Algorithm 2 below attempts to combine the merits of both Algorithm 0 and Algorithm 1. Let $S_{j}$ denote the set of possible current states right after the first $j \mathrm{I} / \mathrm{O}$ pairs of $Q$, i.e., $S_{j}=\delta\left(S_{0}, x_{1} x_{2} \ldots x_{j}\right)$. In Algorithm 2, Algorithm 0 is used first to reduce the size of $S_{j}$ and then Algorithm 1 is used on the current $S_{j}$ with the remaining portion of the $\mathrm{I} / \mathrm{O}$ sequence $Q$.

\section{Algorithm 2}

Given: $\begin{aligned} & F S M M=(S, X, Y, \delta, \lambda), S_{0} \subseteq S, q \leq k, \\ & \mathrm{I} / \mathrm{O} \text { sequence } Q=\left(x_{1} / y_{1}\right)\left(x_{2} / y_{2}\right) \ldots\left(x_{k} / y_{k}\right)\end{aligned}$

Begin:

$j \leftarrow 1 ; / * j$ is the counter for $\mathrm{I} / \mathrm{O}$ pairs $* /$

$S^{\prime} \leftarrow S_{0}$;

while $(j \leq q) / *$ Algorithm 0 up to $Q_{q}^{p} * /$

if $\left(S^{\prime} \neq \varnothing\right)\{$

$S^{\prime \prime} \leftarrow \varnothing$;

for (every $s \in S^{\prime}$ )

if $\left(y_{j}=\lambda\left(s, x_{j}\right)\right) S^{\prime \prime} \leftarrow S^{\prime \prime} \cup \delta\left(s, x_{j}\right)$;

/*redundant states in $S^{\prime \prime}$ are removed*/

endfor

$S^{\prime} \leftarrow S^{\prime \prime} ; j \leftarrow j+1 ;$

\}

else $\quad / * S^{\prime}=\varnothing * /$

endwhile

return (" $N$ is faulty");

while $\left(S^{\prime} \neq \varnothing\right)$

/* Algorithm 1 starting from $S_{q}=S^{*}$ /

randomly choose a state $s$ from $S^{\prime}$;

$S^{\prime} \leftarrow S^{\prime} \mid\{s\} ; j \leftarrow q+1 ;$

$/ * j$ is the counter for I/O pairs */

while $\left(j<k\right.$ AND $\left.y_{j}=\lambda\left(s, x_{j}\right)\right)$ 


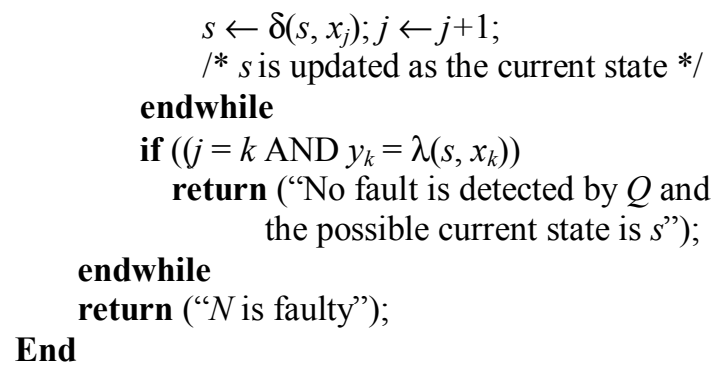

The computational complexity of Algorithm 2 is obtained by combining the results in Theorem 1 and Theorem 2 and it is affected by the selection of a value for variable $q$. Suppose that, in Algorithm 0 part of Algorithm 2, $j_{1}$ is the minimum number of $\mathrm{I} / \mathrm{O}$ pairs needed to eliminate the redundant checking problem and $j_{2}$ is the minimum number of $\mathrm{I} / \mathrm{O}$ pairs needed to reduce the size of possible current states to be one or zero. Obviously, $j_{1}$ is smaller than $j_{2}$. Clearly,

if $q<j_{1}$, the redundant checking problem remains;

if $q>j_{2}$, Algorithm 2 is the same as Algorithm 0;

if $j_{1} \leq q<j_{2}$, Algorithm 2's performance is at least equal to that of Algorithm 0 . However, since $j_{1}$ and $j_{2}$ are both determined by $Q$ and $M$, it is difficult to determine a proper value for $q$. Also, Algorithm 0 part makes Algorithm 2 unable to determine the possible starting state unless a post-processing is performed.

Algorithm 0 is not efficient enough as it has to check all the states in $S_{0}$ and doesn't provide information about possible starting states. Algorithm 1 's efficiency is influenced by the redundant checking problem. Algorithm 2 attempts to eliminate the redundant checking problem by combining the merits of both Algorithm 0 and Algorithm 1 but the effort is limited as it introduces a variable $q$ for which it is difficult to find an appropriate value. Also, Algorithm 2 cannot determine the possible starting state. Algorithm 3 presented below overcomes the drawbacks of these three algorithms:

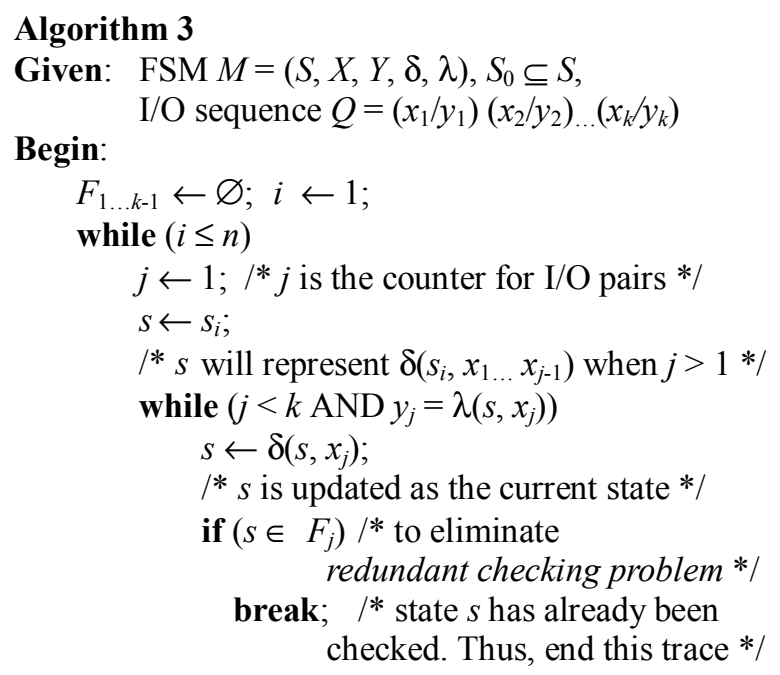

break; /* state $s$ has already been checked. Thus, end this trace*/

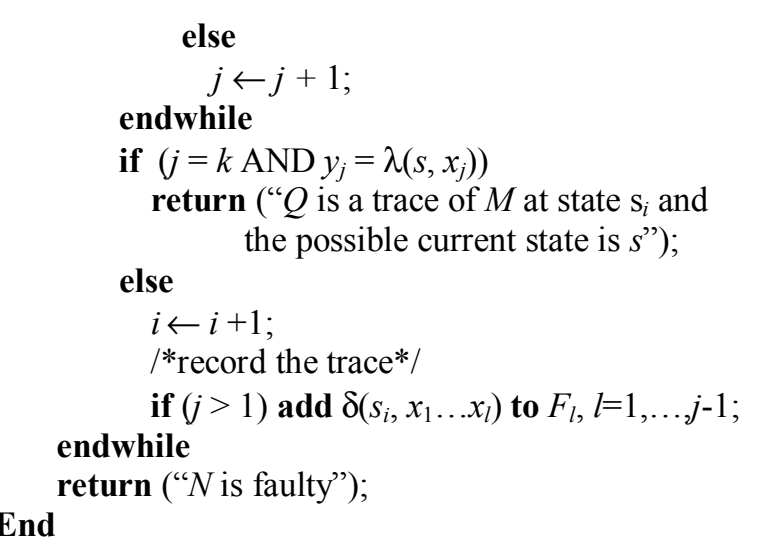

The data structure $F_{1 \ldots k-1}$ is used to record the tracing history and therefore to avoid the redundant checking problem. If $\delta\left(s_{i}, x_{1} x_{2} \ldots x_{j}\right) \in F_{j}, 1 \leq j \leq k$, then $\lambda\left(\delta\left(s_{i}, x_{1} x_{2} \ldots x_{j}\right), x_{j+1} \ldots x_{k}\right)$ has already been checked and $y_{j+1 . .} y_{k} \neq \lambda\left(\delta\left(s_{i}, x_{1} x_{2} \ldots x_{j}\right), x_{j+1} \ldots x_{k}\right)$. So, $Q_{j}^{s}$ will not need to be checked and the checking started from state $s_{i}$ will stop. After checking a state $s_{i}$, if $s_{i}$ is not an eligible starting state, Algorithm 3 adds the trace history starting from $s_{i}$ into $F_{1 \ldots j-1}$. If $s_{i}$ is compatible with $Q$, the possible starting state $s_{i}$ and its corresponding trace are determined.

Theorem 3 For a given state $s_{i}$ of $M$ and an $\mathrm{I} / \mathrm{O}$ sequence $Q=x_{1} \ldots x_{k} / y_{1} \ldots y_{k}$, let $c_{i}^{\prime}(Q)$ denote the largest number $j(1 \leq j \leq \mathrm{k})$ such that (1) $y_{1} \ldots y_{j-1}=\lambda\left(s_{i}\right.$, $\left.x_{1} \ldots x_{j-1}\right)$ and $\lambda\left(\delta\left(s_{i}, x_{1} \ldots x_{j-1}\right), x_{j}\right) \neq y_{j}$; (2) for every $l$ (1 $\leq l \leq j-1), \delta\left(s_{i}, x_{1} \ldots x_{l}\right) \notin F_{l}$. If the $r^{\text {th }}$ state checked, $s_{r}$, is the first state of $M$ such that $Q$ is a trace of $M$ at $s_{r}$, then the computational complexity of Algorithm 3:

$C_{3}\left(M, S_{0}, Q\right)=\sum_{i=1}^{r} c_{i}^{\prime}(Q)$;

if $N$ is faulty, then $C_{3 \text { worst }}\left(M, S_{0}, Q\right)=\sum_{i=1}^{n} c_{i}^{\prime}(Q)$; if $r=1, C_{3 \text { best }}\left(M, S_{0}, Q\right)=c_{1}^{\prime}(Q)$.

Proof: Compared to Algorithm 1, the checking procedure of Algorithm 3 on state $s_{i}$ will stop when it encounters a mismatch with $Q$, the whole $Q$ has been checked compatible, or $\delta\left(s_{i}, x_{1} x_{2} \ldots x_{j}\right) \in F_{j}$. Similar to Theorem 2 , let $c_{i}^{\prime}(Q)$ denote the largest number $j(1 \leq j$ $\leq k$ ) before checking on $s_{i}$ terminates. If $s_{r}$ is the first state of $M$ such that $Q$ is the trace of $M$ at $s_{r}$, then the total number of comparisons needed is $\sum_{i=1}^{r} c_{i}^{\prime}(Q)$.

As the states compatible with $Q$ are randomly dispersed in $S_{0}$, the process of sequentially checking the states within $S_{0}$ until a state compatible with $Q$ is found can be modeled as the Sampling without Replacement Model [3].

Theorem 4 According to the Sampling without Replacement Model, assume that there are $m$ states in $S_{0}$ which are compatible with $Q$, and $r(1 \leq r \leq n-m+1)$ states are randomly selected from $S_{0}$. Then, the 
probability that the $r^{\text {th }}$ state is the first state which is checked to be compatible with $Q$ is given by

$$
P_{r}(m)= \begin{cases}\frac{m}{n} & (r=1) ; \\ \frac{m(n-m)(n-m+1) \ldots(n-m-r+2)}{n(n-1) \ldots(n-r+1)} & (2 \leq r \leq n-m+1) .\end{cases}
$$

Proof: Each different arrangement of states selected from $S_{0}$ is called a permutation. Suppose that $r$ states are selected one at a time and removed from $S_{0}(1 \leq r$ $\leq n-m+1)$. Then each possible outcome of this selection will be a permutation of $r$ states from $S_{0}$, and the total number of these permutations will be $P_{n, r}=$ $n(n-1) \ldots(n-r+1)$ [3]. $P_{n, r}$ is called the number of permutations of $n$ elements taken $r$ at a time. Thus, if $r$ $=1$, the number of permutations is $n$; if $2 \leq r \leq n-m+1$, the number of permutations, in which the $r^{\text {th }}$ state is the first state compatible with $Q$, is $m P_{n-m, r-1}=m(n-$ $m)(n-m-1) \ldots(n-m-r+2)$. Then the probability of permutation that the $r^{\text {th }}$ state is the first state compatible with $Q$ is: if $r=1, P_{r}(m)=m / P_{n, r}=m / n$;

if $2 \leq r \leq n-m+1, P_{r}(m)=m P_{n-m, r-1} / P_{n, r}=$

$\frac{m(n-m)(n-m+1) \ldots(n-m-r+2)}{n(n-1) \ldots(n-r+1)}$.

Theorem 5 Suppose there are $m(0 \leq m \leq n)$ states in $S_{0}$ which are compatible with $Q$. Let $P_{r}(m)$ denote the probability that the $r^{\text {th }}$ state is the first state which is compatible with $Q$. The average computational complexity of Algorithm 3 is $A_{3}=$

$\sum_{r=1}^{n-m+1} P_{r}(m) C_{3}\left(M, S_{0}, Q\right)=\sum_{\mathrm{r}=1}^{\mathrm{n}-\mathrm{m}+1}\left(P_{r}(m) \sum_{i=1}^{r} c_{i}^{\prime}(Q)\right)$.

Proof: The average computational complexity of Algorithm 3 is the sum of the number of comparisons multiplied by its corresponding probability [9].

Example 4. Assume $n=4, m=1$, when $r=1, C_{3}=4$; when $r=2, C_{3}=5$; when $r=3, C_{3}=7$; when $r=4, C_{3}$ $=9$ where $C_{3}$ stands for $C_{3}\left(M, S_{0}, Q\right)$; Then, $A_{3}=1+5 / 4+7 / 4+9 / 4=25 / 4$ (see Table 1$)$.

Table 1. Average computational complexity analysis

\begin{tabular}{|c|c|c|c|}
\hline$r$ & $C_{3}$ & $P_{r}(m=1)$ & $P_{r}(m=1) C_{3}$ \\
\hline 1 & 4 & $1 / 4$ & 1 \\
\hline 2 & 5 & $1 * 3 / 4 * 3=1 / 4$ & $5 / 4$ \\
\hline 3 & 7 & $1 * 3 * 2 / 4 * 3 * 2=1 / 4$ & $7 / 4$ \\
\hline 4 & 9 & $1 * 3 * 2 * 1 / 4 * 3 * 2 * 1=1 / 4$ & $9 / 4$ \\
\hline
\end{tabular}

In general, the number of states compatible with $Q$ may be zero, or more. If it is zero, it means that none of the states in $S_{0}$ is compatible with $Q$ and $N$ is faulty; if it is one or more than one, it means that the given $Q$ is insufficient to determine whether $N$ is faulty.

The general case can be simplified to the case in which the number of states which are compatible with $Q$ is either one or zero. This stems from the fact that the essence of passive fault detection is to detect the existence of faults in $N$. If there is one or more states compatible with $Q$, it implies that the given $Q$ is insufficient to come to a conclusion. Additional $\mathrm{I} / \mathrm{O}$ sequence $\Delta Q$ is needed to continue with the fault detection. Thus, let $Q^{\prime}=Q+\Delta Q$ denote the $\mathrm{I} / \mathrm{O}$ sequence concatenating $Q$ to $\Delta Q$. Thus, the new set ( $M$, $S_{0}, Q$ ) contains at most one state that is compatible with $Q^{\prime}$. Let $P_{r}(m=1)$ denote the probability that there is one compatible state in $S_{0}$ and it appears at the $r^{\text {th }}(1$ $\leq r \leq n)$ selection. So, $P_{r}(m=1)=1 / n$.

Theorem 6: If there is only one state in $S_{0}$ that is compatible with $Q$, the average computational complexity of Algorithm 3 is

$A_{3}=\frac{1}{n} \sum_{r=1}^{n} C_{3}\left(M, S_{0}, Q\right)=\frac{1}{n} \sum_{\mathrm{r}=1}^{\mathrm{n}}\left(\sum_{i=1}^{r} c_{i}^{\prime}(Q)\right)$.

Proof: The average computational complexity of Algorithm 3 is the sum of the number of comparisons multiplied by its corresponding probability.

\subsection{Comparison of the Algorithms}

The computational complexities of the three algorithms given in the previous subsections are summarized in Table 2 .

Table 2. Computational complexity

\begin{tabular}{|l|l|}
\hline Type of algorithm & Computational complexity \\
\hline Algorithm 0 & $C_{1}=\sum_{j=1}^{k}\left|S_{j-1}\right|$ \\
\hline Algorithm 1 & $C_{2}\left(M, S_{0}, Q\right)=\sum_{i=1}^{r} c_{i}(Q)$ \\
\hline Algorithm 3 & $C_{3}\left(M, S_{0}, Q\right)=\sum_{i=1}^{r} c_{i}^{\prime}(Q)$ \\
\hline
\end{tabular}

- $k$ is the length of $Q,\left|S_{j}\right|$ is the number of states in the set of possible current states,

- $r$ is the number of states checked before a state compatible with $Q$ is found,

- $c_{i}(Q)$ is the largest number $j(1 \leq j \leq \mathrm{k})$ such that $y_{1} \ldots y_{j-1}=\lambda\left(s_{i}, x_{1} \ldots x_{j-1}\right)$ and $\lambda\left(\delta\left(s_{i}, x_{1} \ldots x_{j-1}\right), x_{j}\right) \neq y_{j}$

- $c_{i}^{\prime}(Q)$ is the largest number $j(1 \leq j \leq \mathrm{k})$ such that $y_{1} \ldots y_{j-1}=\lambda\left(s_{i}, x_{1} \ldots x_{j-1}\right)$ and $\lambda\left(\delta\left(s_{i}, x_{1} \ldots x_{j-1}\right), x_{j}\right) \neq y_{j}$ after eliminating the redundant checking problem

Below, we compare the computational complexities of Algorithm 0 and Algorithm 3 when the number of states in $S_{0}$ which are compatible with $Q$ is one or zero. In Algorithm 0, once the set $\left(M, S_{0}, Q\right)$ is fixed, the number of comparisons needed is determined and does not change during its application. On the other hand, the performance of Algorithm 3 is affected by the number of states in $S_{0}$ which are compatible with $Q$ (see Theorem 4). Algorithm 0 and Algorithm 3 represent different perspectives on tracing order in passive fault detection. Algorithm 0 checks all the states in the set of possible current states with one $\mathrm{I} / \mathrm{O}$ 
pair in $Q$ at a time whereas Algorithm 3 selects one starting state from $S_{0}$ and exhausts all the possible transitions starting from this state according to the $\mathrm{I} / \mathrm{O}$ sequence $Q$. If there is no state in $S_{0}$ which is compatible with $Q$, both Algorithm 0 and Algorithm 3 need to check the entire trace from every state in $S_{0}$ and thus they perform equally. That is $\sum_{i=1}^{n} c_{i}^{\prime}(Q)=\sum_{j=1}^{k}\left|S_{j-1}\right|$. If there is only one state $s$ in $S_{0}$ which is compatible with $Q$, then the total number of comparisons made by Algorithm 0 is $\sum_{j=1}^{k}\left|S_{j-1}\right|$ whereas the total number of comparisons made by Algorithm 3 is $\sum_{i=1}^{r} c_{i}^{\prime}(Q)(r \leq n)$. Clearly, $\sum_{i=1}^{r} c_{i}^{\prime}(Q) \leq$ $\sum_{i=1}^{n} c_{i}^{\prime}(Q)=\sum_{j=1}^{k}\left|S_{j-1}\right|,(r \leq n)$ where the $r^{\text {th }}$ state checked is the state compatible with $Q$.

Thus, Algorithm 3 always performs at least as well as Algorithm 0. The equality in their computational complexities occurs when $r=n$.

Based on the computational complexities of the algorithms presented above, several assertions can be made on their performance in different conditions.

When $N$ is not determined to be faulty:

i) if there is no redundant checking problem,

the performance of Algorithm 1 will be the same as that Algorithm 3 and be at least equal to that of Algorithm 0; and the performance of Algorithm 2 will be between those of Algorithms 0 and 1 .

ii) if there is redundant checking problem, the performance of Algorithm 3 will be at least equal to those of Algorithms 0 and 1; and

it is not possible to compare the performances of Algorithm 0, Algorithm 1, and Algorithm 2 analytically due to the redundant checking problem.

When $N$ is determined to be faulty:

i) if there is no redundant checking problem, the performances of all the algorithms will be the same.

ii) if there is redundant checking problem, the performance of Algorithm 3 will be equal to that of Algorithm 0; the performances of Algorithms 1 and 2 will at most be equal to that of Algorithm 0; and it is not possible to compare the performances of Algorithms 0 and 2 analytically.

\section{Experimental Evaluation}

An experimental evaluation is made to compare the average computational complexity of the algorithms and to verify the validity of the assertions drawn above when $m=0$ or 1 . In the experiment, we use a set of randomly generated FSMs. This set consists of FSMs with different number of states $\left(\left|S_{0}\right|=|S|\right)$, set $X$ of inputs and set $Y$ of outputs. We select 5 configurations in the form of $\left(\left|S_{0}\right|,|X|,|Y|\right)$, namely $(5,3,3),(10,4,4)$, $(15,4,4),(20,5,5),(30,10,10)$. For each configuration, we generate 5 FSMs correspondingly. For each FSM $M$, two cases are considered.

In Case I, called correct implementation, there is exactly only one state in $S_{0}$ that is compatible with $Q$ $(m=1)$. In Case II, called faulty implementation, there is no state in $S_{0}$ that is compatible with $Q(m=0)$ and "faulty" is expected to be reported. We create a faulty specification $M^{\prime}$ from $M$ by altering either the output or next state of a (randomly) selected transition. In Case I (Case II), for every state $s$ of $M\left(M^{\prime}\right)$, we generate three random $\mathrm{I} / \mathrm{O}$ sequences of length $\left|S_{0}\right| *|X| * 2,\left|S_{0}\right| *|X| * 4,\left|S_{0}\right| *|X| * 10$ respectively, starting from $s$; and when generating each I/O sequence $Q$, we randomly select a transition of the current state of $M$ $\left(M^{\prime}\right)$ and repeat this at the next state.

Then, we apply all four algorithms to the FSMs in these two cases and record the results. Table 3 shows the number of comparisons (between the actual output $y_{j}$ and the expected output $\left.\lambda\left(s, x_{j}\right)\right), 1 \leq j \leq k, s \in S$, for each of the four algorithms. We see from Table 3 that,

- Algorithm 1, in Case I, has better performance than Algorithm 0 in average case and best case, but not in worst case. Also, in Case II, Algorithm 1 cannot beat Algorithm 0;

- Algorithm 2 performs the same as the Algorithm 0 because the number of states in the set of possible current states shrinks to one or zero in the "Algorithm 0" part of Algorithm 2.

- Algorithm 3, in Case I, needs fewer comparisons to find the compatible state and performs better than Algorithm 0; while in Case II, these two algorithms perform the same.

Experimental results confirm the assertions we present in Section 3 and show that Algorithm 3 performs best among these four algorithms when there is one state in $S_{0}$ compatible with $Q$ (Case I).

\section{Conclusions}

In this paper, we proposed a new approach to Finite State Machine-based passive fault detection. Compared with the former approach in [4], the proposed approach (Algorithm 3) has better performance and provides more information during testing. Specifically, Algorithm 3 provides more information about possible starting state and possible trace compatible with the observed sequence $Q$ and performs better in situations where there is only one state in $S_{0}$ that is compatible with $Q$. The results of both theoretical and experimental evaluations confirm this improvement over the approach in [4]. 
Table 3. Experimental results

\begin{tabular}{|c|c|c|c|c|c|c|c|c|}
\hline \multirow{7}{*}{ Algorithm 0} & & & \multicolumn{3}{|c|}{ Case I $: m=1$} & \multicolumn{3}{|c|}{ Case II $: m=0$} \\
\hline & $|Q|$ & $\left|S_{0}\right|$ & best & worst & average & best & worst & average \\
\hline & 60 & 5 & 64 & 74 & 65.5 & 6 & 58 & 17.8 \\
\hline & 160 & 10 & 169 & 175 & 171.3 & 11 & 162 & 31.7 \\
\hline & 240 & 15 & 254 & 262 & 258.0 & 16 & 252 & 42.8 \\
\hline & 400 & 20 & 419 & 429 & 423.0 & 21 & 399 & 44.7 \\
\hline & 1200 & 30 & 1229 & 1236 & 1231.9 & 31 & 1122 & 73.0 \\
\hline \multirow[t]{6}{*}{ Algorithm 1} & $|Q|$ & $\left|S_{0}\right|$ & best & worst & average & best & worst & average \\
\hline & 60 & 5 & 60 & 74 & 62.3 & 7 & 58 & 18.7 \\
\hline & 160 & 10 & 160 & 172 & 165.5 & 11 & 202 & 36.9 \\
\hline & 240 & 15 & 240 & 263 & 247.7 & 16 & 252 & 46.1 \\
\hline & 400 & 20 & 400 & 432 & 409.3 & 21 & 744 & 52.9 \\
\hline & 1200 & 30 & 1200 & 1235 & 1215.0 & 31 & 1531 & 83.7 \\
\hline \multirow{6}{*}{$\begin{array}{l}\text { Algorithm } 2 \\
q=5 \text { ( } q \text { is } \\
\text { defined in } \\
\text { Algorithm 2) }\end{array}$} & $|Q|$ & $\left|S_{0}\right|$ & Best & worst & average & best & worst & average \\
\hline & 60 & 5 & 64 & 74 & 65.5 & 6 & 58 & 17.8 \\
\hline & 160 & 10 & 169 & 175 & 171.3 & 11 & 162 & 31.7 \\
\hline & 240 & 15 & 254 & 262 & 258.0 & 16 & 252 & 42.8 \\
\hline & 400 & 20 & 419 & 429 & 423.0 & 21 & 399 & 44.7 \\
\hline & 1200 & 30 & 1229 & 1236 & 1231.9 & 31 & 1122 & 73.0 \\
\hline \multirow[t]{6}{*}{ Algorithm 3} & $|Q|$ & $\left|S_{0}\right|$ & Best & worst & average & best & worst & average \\
\hline & 60 & 5 & 60 & 65 & 61.9 & 6 & 58 & 17.8 \\
\hline & 160 & 10 & 160 & 171 & 164.3 & 11 & 162 & 31.7 \\
\hline & 240 & 15 & 240 & 262 & 247.9 & 16 & 252 & 42.8 \\
\hline & 400 & 20 & 400 & 427 & 409.6 & 21 & 399 & 44.7 \\
\hline & 1200 & 30 & 1200 & 1234 & 1215.3 & 31 & 1122 & 73.0 \\
\hline
\end{tabular}

\section{Acknowledgments}

This work was supported in part by grants from Natural Sciences and Engineering Research Council of Canada, and Ontario Centers of Excellence.

\section{References}

[1] B. Alcalde, A. Cavalli, D. Chen, D. Khuu and D. Lee (2004) "Network Protocol System Passive Testing for Faulty Management - a Backward Checking Approach," Proc. of IFIP FORTE'04, LNCS, vol. 3235, pp.150-166.

[2] D. Chen, J. Wu, and T.L. Chu (2003) "An Enhanced Passive Testing Tool for Network Protocols," Proc. of ICCNMC'03, pp.513-516.

[3] M.H. DeGroot and M.J. Schervish. Probability and Statistics. Boston: Addison-Wesley, 2002.

[4] D. Lee, A.N. Netravali, K.K. Sabnani, B. Sugla, and A. John (1997) "Passive Testing and Applications to Network Management," Proc. of ICNP'97, pp.113-122.

[5] D. Lee, D. Chen, R. Hao, R.E. Miller, J. Wu and $X$. Yin (2002) "A Formal Approach for Passive Testing of Protocol Data Portions," Proc. of ICNP'02, pp.122-131.
[6] D. Lee, D. Chen, R. Hao, R.E. Miller, J. Wu and X. Yin (2006) "Network Protocol System Monitoring - A Formal Approach with Passive Testing," IEEE/ACM Transactions on Networking, vol.14, pp.424-437.

[7] R.E. Miller (1998) "Passive Testing of Networks Using a CFSM Specification," Proc. of IPCCC'98, pp.111-116.

[8] R.E. Miller and K.A. Arisha (2001) "Fault Identification in Networks by Passive Testing," Proc. of $34^{\text {th }}$ Annual Simulation Symposium, pp.277-284.

[9] R. Neapolitan and K. Naimipour. Foundations of Algorithms Using C ++ Pseudocode, $3^{\text {rd }}$ Edition. Sudbury, Mass.: Jones \& Bartlett Publishers, 2003.

[10]M. Tabourier and A. Cavalli (1999) "Passive testing and application to the GSM-MAP protocol," Information and Software Technology, vol. 41, pp.813-821.

[11] J. Wu, Y. Zhao, and X. Yin (2001) "From Active to Passive: Progress in Testing of Internet Routing Protocols," Proc. of FORTE' 01, pp.101-118.

[12] Y. Zhao, X. Yin, and J. Wu (2001) "OnLine Test System, an Application of Passive Testing in Routing Protocols," Proc. of ICN'01, pp.190-195. 